\title{
A New Explanation for the Color Variety of Photons
}

\author{
Gh. Saleh ${ }^{1}, M . J$. Faraji $^{1}, R$. Alizadeh $^{1}$, and $A$. Dalili $^{1}$ \\ ${ }^{1}$ Saleh Research Centre, Shiraz, Fars, Iran
}

\begin{abstract}
This new explanation is based on Wave-Particle Duality and Newtonian Laws and represents a unique definition of a three-dimensional motion for the photon, whose dual behavior is partly explained by the double-slit experiment of Thomas Young, who represents the photon's motion as a wave, and by the Photoelectric effect, in which the photon is considered as a particle. However, for scientists, the photon's true motion is unclear. In this article, we define a new type of motion for photons to solve both this ambiguity and the difficulty of presenting a three-dimensional trajectory for the photon's motion, and present a new formula to calculate its energy. In addition, because we believe in the helical motion of photons, where $\mathrm{r}$ is the gyroradius, we believe that their color is an effect of the order of magnitude of $r$. We present real examples that prove our energy formula.
\end{abstract}

\section{Introduction}

"It seems as though we must use sometimes the one theory and sometimes the other, while at times we may use either. We are faced with a new kind of difficulty" When Einstein said [1] this, he was curious to know more about the photon's behavior.

Actually, when a photon is emitted by an excited electron, it has two different motions: a rotation along and around a straight axis at light speed (v), and a threedimensional trajectory combining both rectilinear and orbital trajectories. However, a side view of this movement still shows a sine wave (Fig. 1).

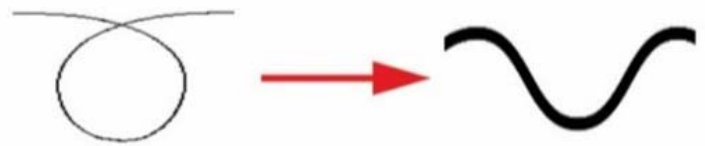

Fig. 1. Side view of a photon's motion appears as a sine wave.

If we imagine the electron as a rotating fountain that turns around itself, the photon is diffused in the same way that the fountain projects the drop of water, and the photon will follow the electron's rotation around its nucleus. Therefore, the photon, in addition to its linear trajectory, also turns around an assumed axis, ultimately creating a three-dimensional trajectory. To approach an understanding of this, we consider the example of a released spring. That is to say, the photon's trajectory is a result of the combination of two motions: the rectilinear one and a perpendicular circulation around the same axis (z).

Actually, due to the intensity of the energy released during the emission, the photon rotates both around its own axis (like the Earth's rotation), and around an external point (similar to the annual motion of the Earth) while continuing its rectilinear trajectory. Therefore, one turn of the photon around the external point is equal to the wavelength of a sinusoidal wave. Thus, the number of turns of the photon in a second is called its "Frequency" or " $\vartheta$ ". Therefore, the energy of photons consists of two separate parts: the first is rotational energy, which depends on its distance from the center (r). The second depends on the number of turns per unit of time.

We believe that the photon has constant mass (m); therefore, its kinetic energy with the consideration of classic mechanics [2] is equal to:

$E_{\text {rot }}=1 / 2 I \omega^{2}$

where (I) is the moment of inertia, and $(\omega)$ is angular speed.

Moreover, because a photon has a constant and small mass (m), it also maintains a constant distance from the center of rotation (r) [2]; therefore,

$I=m r^{2}$

As a result, its rotational kinetic energy is equal to: $E_{\text {rot }}=1 / 2 m r^{2} \omega^{2}$

In this paper, we denote $E_{\text {rot }}$ as the "inner part of the kinetic energy"

The energy of the photon also depends on the number of turns in a unit of time, or its frequency, which is expressed by the Planck formula [3]:

$E_{1}=h \vartheta$

where h is Planck's constant and $\vartheta$ is the frequency.

In this paper, we denote $E_{1}$ as "the wave part of the kinetic energy" (Fig. 2). 


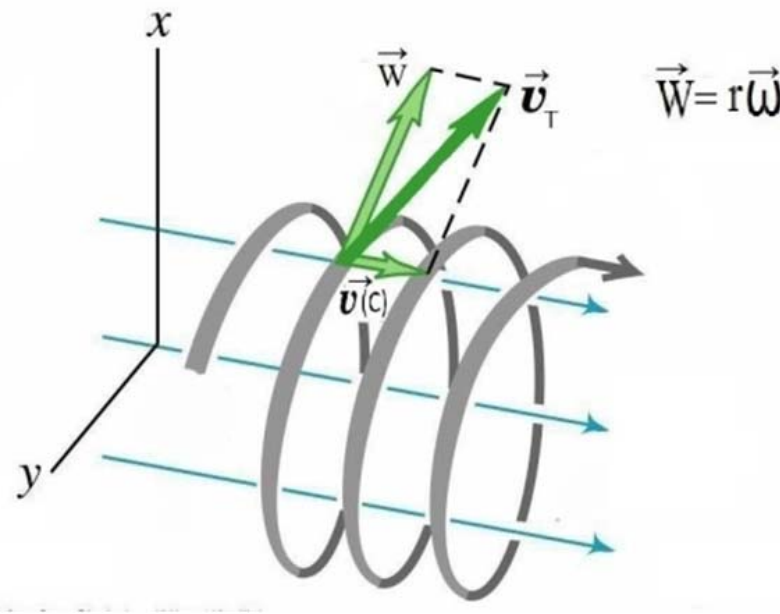

Fig. 2. Diagram of 3D motion of photon.

As a result, the total kinetic energy of a photon is equal to the sum of both the wave and the inner parts:

$E_{\text {Tot }}=E_{1}+E_{\text {rot }}=h \vartheta+1 / 2 m r^{2} \omega^{2}$

However, when we consider the photon as a particle it will eventually displaced along one axis (for example $\mathrm{Z})$ with a velocity of $(\mathrm{V})$; therefore, its kinetic energy is equal to [4]:

$\mathrm{E}=\int F \cdot d z$

$F=\frac{d P}{d t}=\frac{d(m v)}{d t}$

$E=\int \frac{d(m v)}{d t} d z=\int d(m v) \frac{d z}{d t}=m \int v d v$

$E=1 / 2 m v^{2}$

Consequently,

$E=1 / 2 m v^{2}=h \vartheta+1 / 2 m r^{2} \omega^{2}$

$h \vartheta=1 / 2 m v^{2}-1 / 2 m r^{2} \omega^{2}$

$h \vartheta=1 / 2 m\left(v^{2}-r^{2} \omega^{2}\right)$

where $\mathrm{m}$ is the photon's mass, $\mathrm{v}$ its velocity in a straight line, $\mathrm{r}$ its gyroradius, $\mathrm{h}$ the Planck constant, $\vartheta$ its frequency, and $\omega$ its angular velocity. In this formula, $v$ is the velocity of the photon along the imaginary axis and equals the speed of light $C$, and $\omega$ has a constant value in the consumable part.

The $1 / 2 m r^{2} \omega^{2}$ represents the inner part of the kinetic energy and $\mathrm{h} \vartheta$ represents the wave part. Because $\mathrm{m}, \mathrm{h}, \omega$ and $\mathrm{v}$ are constant in one area, Formula (12) represents that the energy of photons is dependent on the frequency $(\vartheta)$ and gyroradius (r). However, the total kinetic energy is constant. An increase in frequency will decrease the gyroradius, and vice versa.

Imagine that a photon is traversing a vacuum with a constant velocity " $\mathrm{V}=\mathrm{C}$ ". In this condition, Equation (10) is

$E=1 / 2 m c^{2}$

$1 / 2 m c^{2}=h \vartheta+1 / 2 m r^{2} \omega^{2}$

$1 / 2 m c^{2}-1 / 2 m r^{2} \omega^{2}=h \vartheta$

In addition, because $\mathrm{C}$ and $\mathrm{m}$ are constant, the photon's energy is constant. If this photon has a frequency such as $\vartheta_{1}$ its gyroradius will be $r_{1}$. If its frequency equals $\vartheta_{2}$, its gyroradius will be $r_{2}$, and $1 / 2 m c^{2}$ always has a constant value.

\section{The Colorfulness of Photons}

The visible spectrum is a concept that has attracted much attention. We believe that all photons are the same; they have a constant mass, and that their spectral distinction is due to the gyroradius. Changes in the gyroradius induce color changes. In fact, an increase in the gyroradius reciprocally means the lengthening of the wavelength. For example, the long wavelength $(\lambda)$ of the red spectrum is a result of a high value of the gyroradius $r$, while the blue spectrum is the opposite (Fig. 3).

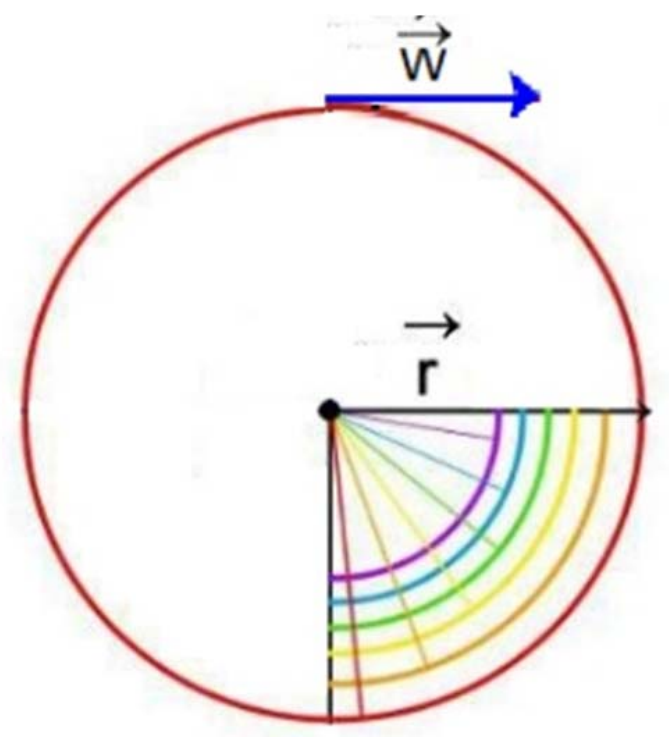

Fig. 3. Diagram of gyroradius.

We therefore consider a photon as a particle with a constant mass that generates different spectra following the variations in its gyroradius, and, indeed, that color is an effect of the order of magnitude of $r$.

\section{Domain of $\vartheta, \lambda, r$}

We assume that the mass of the photon is $m=1.637 \times$ $10^{-35} \mathrm{~kg}$ and that the approximate value of its angular velocity is equal to $=1.67 \times 10^{15} \mathrm{rad} / \mathrm{s}$. The gyroradius can be calculated for three spectra: violet $\lambda=380$ n.m., yellow $\lambda=580$ n.m. and red $\lambda=750$ n.m., using equation (15):

$$
\begin{aligned}
& \frac{1}{2} m c^{2}-\frac{1}{2} m r^{2} \omega^{2}=h \vartheta \\
& m c^{2}-m r^{2} \omega^{2}=\frac{2 h c}{\lambda} \Rightarrow m r^{2} \omega^{2}=m c^{2}-\frac{2 h c}{\lambda} \Rightarrow r^{2}=\frac{c^{2}}{\omega^{2}}-\frac{2 h c}{\lambda m \omega^{2}} \\
& \Rightarrow r=\sqrt{\frac{c^{2}}{\omega^{2}}-\frac{2 h c}{\lambda m \omega^{2}}}
\end{aligned}
$$




$$
\begin{aligned}
& \lambda_{1}=380 \mathrm{~nm} \Rightarrow \vartheta=780 \times 10^{12} \mathrm{~Hz} \Rightarrow r=97.9 \mathrm{~nm} \\
& \lambda_{2}=580 \mathrm{~nm} \Rightarrow \vartheta=517 \times 10^{12} \mathrm{~Hz} \Rightarrow r=131.2 \mathrm{~nm}: \\
& \lambda_{3}=750 \mathrm{~nm} \Rightarrow \vartheta=400 \times 10^{12} \mathrm{~Hz} \Rightarrow r=143.6 \mathrm{~nm}
\end{aligned}
$$

\section{Red:}

$$
\begin{aligned}
& \vartheta=400 \times 10^{12}{ }_{\mathrm{Hz}} \\
& r=143.6 \times 10^{-9} \mathrm{~m}
\end{aligned}
$$

$1 / 2 m c^{2}-1 / 2 m r^{2} \omega^{2}$

$$
\begin{aligned}
& =1 / 2 \times 1.637 \times 10^{-35} \\
& \times\left(2.998 \times 10^{8}\right)^{2}-1 / 2 \times 1.637 \\
& \times 10^{-35} \times\left(143.6 \times 10^{-9}\right)^{2} \\
& \times\left(1.67 \times 10^{15}\right)^{2}=2.65 \times 10^{-19} j
\end{aligned}
$$

$h \vartheta=6.626 \times 10^{-34} \times 400 \times 10^{12}=2.65 \times 10^{-19} j$

\section{Yellow:}

$$
\begin{aligned}
& \vartheta=517 \times 10^{12}{ }_{\mathrm{Hz}} \\
& r=131.2 \times 10^{-9}{ }_{\mathrm{m}}
\end{aligned}
$$

$$
1 / 2 m c^{2}-1 / 2 m r^{2} \omega^{2}
$$

$$
\begin{aligned}
& =1 / 2 \times 1.637 \times 10^{-37} \\
& \times\left(2.998 \times 10^{8}\right)^{2}-1 / 2 \times 1.637 \\
& \times 10^{-37} \times\left(131.2 \times 10^{-9}\right)^{2} \\
& \times\left(1.67 \times 10^{15}\right)^{2}=3.42 \times 10^{-19} j
\end{aligned}
$$

$h \vartheta=6.626 \times 10^{-34} \times 517 \times 10^{12}=3.42 \times 10^{-19} j$

Violet:

$$
\begin{aligned}
\vartheta & =780 \times 10^{12} \mathrm{~Hz} \\
r & =97.9 \times 10^{-9}{ }_{\mathrm{m}}
\end{aligned}
$$

$1 / 2 m c^{2}-1 / 2 m r^{2} \omega^{2}$

$$
\begin{aligned}
& =1 / 2 \times 1.637 \times 10^{-37} \\
& \times\left(2.998 \times 10^{8}\right)^{2}-1 / 2 \times 1.637 \\
& \times 10^{-37} \times\left(97.9 \times 10^{-9}\right)^{2} \\
& \times\left(1.67 \times 10^{15}\right)^{2}=5.17 \times 10^{-19} j
\end{aligned}
$$

$h \vartheta=6.626 \times 10^{-34} \times 780 \times 10^{12}=5.17 \times 10^{-19} j$

Given the main formula of the Saleh Theory (15) the gyroradius change diagram (Table 1) and domain for $\vartheta, \lambda$, and $\mathrm{r}$ is defined as follow:

$10^{15} \mathrm{~Hz} \geq \vartheta \geq 0_{\mathrm{HZ}}$

$270_{n \cdot m} \leq \lambda \leq \infty$

$0_{n . m} \leq r \leq 180_{n . m}$
Table 1. Gyroradius change diagram according to Saleh Theory.

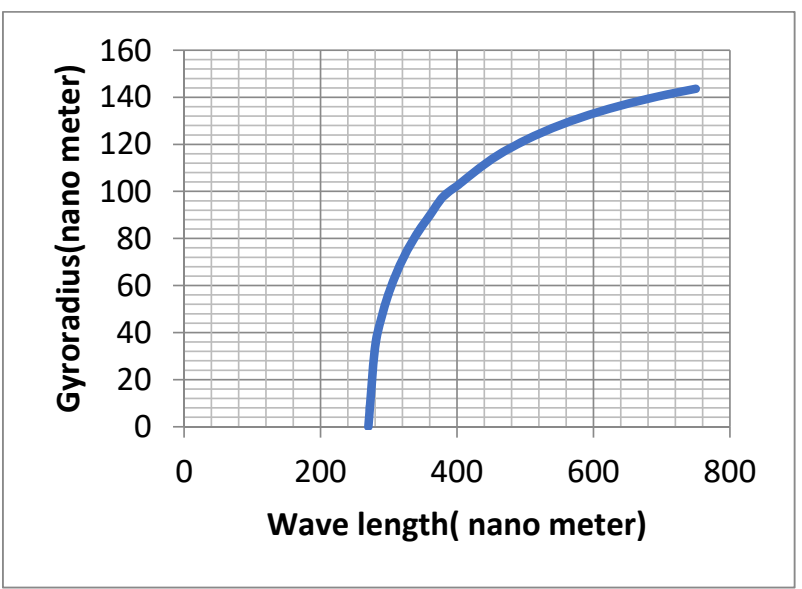

\section{Example}

For example, when the frequency of a photon is $\vartheta_{1}=$ $400_{T H z}$, its gyroradius will be $r_{1}=143.6_{n . m}$ and the spectrum appears red. When a photon's frequency is $\vartheta_{2}=780_{\mathrm{THz}}$, its radius will be $r_{2}=97.9_{n . m}$ and its spectrum will appear violet.

Actually, because the red photon has a different inner kinetic energy from the violet one, our eyes interpret the differences in internal energy such that we perceive them as different colors. This is so important that in our daily life that all warning lights are red, not violet. Because the red spectrum has more inner kinetic energy than the violet one, a red spectrum with the same intensity is visible from a greater distance. If we consider only the wave part of the kinetic energy, according to the Planck and Einstein energy formulae $\left(E=m c^{2}=h \vartheta\right)[3,5]$ the red spectrum has less energy than violet one. Moreover, how can a spectrum with low energy (red spectrum) traverse a greater distance than a violet one with high energy?

\section{This is a Contradiction!}

Saleh Theory states that the red spectrum has the same total kinetic energy as the violet and that they have only one important difference; the inner part of the kinetic energy makes the red spectrum more visible than the violet one from a further distance (Fig. 4).

For a better understanding, we explain another contradiction: Everyone knows that the red spectrum is warm and the blue one is cold [6]. In addition, in physics, being warm means having more energy [7]. This sense and understanding are actually the realization of the internal energy of these spectra. According to the energy formula of Saleh Theory as explained in (8), the red spectrum has a larger gyroradius than does the blue one; thus, it has a higher internal energy. Although their total energies are equal, we receive more internal energy from the red spectrum than from the blue one and we associate the red spectrum with warmth and the blue one with coldness. 
Now look at the Planck formula $(E=h \vartheta)$ [3] on one hand, and combine it with the Einstein formula $E=m c^{2}$ [5] on the other hand. The red spectrum has a lower frequency than the blue one, and it also has a lower mass; therefore, in this condition, the red spectrum, which all of us believe is warm, has lower energy and lower mass $\left(E=h \vartheta=m c^{2}\right)[3,5]$.

\section{This is an Obvious Contradiction!}

The third reason for the energy formula's proof and the three-dimensional rotational motion of photon: The frequency formula of the Doppler Effect in light is [8]:

$\vartheta_{1}=\vartheta_{2} \sqrt{1-\frac{v^{2}}{c^{2}}}$

that is, the result of Einstein's relativity and the Planck formula. Moreover surely

$$
\vartheta_{1} \neq \vartheta_{2}
$$

If we multiply both sides by Planck's constant (h) we have:

$h \vartheta_{1} \neq h \vartheta_{2} \Rightarrow E_{1} \neq E_{2}$

Therefore, the energy sent from the source is different from the energy that we receive.

Now we focus on redshift; when the source producing the blue spectrum is moving away from us, we perceive it as red [8]. Therefore, we have:

$\vartheta_{b}>\vartheta_{\mathrm{r} \Rightarrow \mathrm{h}} \vartheta_{\mathrm{b}}>\mathrm{h} \vartheta_{\mathrm{r}} \Rightarrow \mathrm{E}_{\mathrm{b}}>\mathrm{E}_{\mathrm{r}}$

where $E_{b}$ is the energy that is emitted, and $E_{r}$ is the energy that we receive, $\vartheta$ is the frequency, $\mathrm{h}$ is Planck's constant and the $\mathrm{b} \& \mathrm{r}$ indexes are reagents of the blue and red spectra.

The energies of the red spectra are lower than those of the blue ones $[3,5]$. On the other hand, because Einstein's formula is $E=m c^{2}$ [5],

$m_{b} c^{2}>m_{r} c^{2} \Rightarrow m_{b}>m_{r}$

The red spectrum that we perceive has a lower mass than blue one that is emitted from the source.

$E_{b}>E_{r} \Rightarrow E_{b}-E_{r}>0$

$m_{b}>m_{r} \Rightarrow m_{b}-m_{r}>0$

Therefore, amounts of mass and energy are destroyed at the same time, which violates the principle of conservation of energy and mass [9].

However, in Saleh Theory, because

$E=$ $h \vartheta+1 / 2 m r^{2} \omega^{2}$ explained in (5), the total energy of the photon is always constant, and the spectral color change from blue to red is a conversion of energy from the wave part $(h \vartheta)$ to the inner part $\left(1 / 2 m r^{2} \omega^{2}\right)$;

$E_{b}=E_{r}$

$h \vartheta_{b}+1 / 2 m r_{b}^{2} \omega^{2}=h \vartheta_{r}+1 / 2 m r_{r}^{2} \omega^{2}$

where $\mathrm{m}, \mathrm{h}$ and $\omega$ are constant values.

It is true that $\vartheta_{b}>\vartheta_{r}$ but because $r_{b}<r_{r}$, always, we have:

$E_{\mathrm{b}}=E_{r}$

\section{Conclusions}

Guided by this theory, we can establish a complete threedimensional vision of the photon's motion, which is justified by Wave-Particle Duality and Newtonian Laws.
This vision explains how the variety of light spectra are due to different gyroradius of photon during its trajectory around an imaginary axis (Fig. 4).

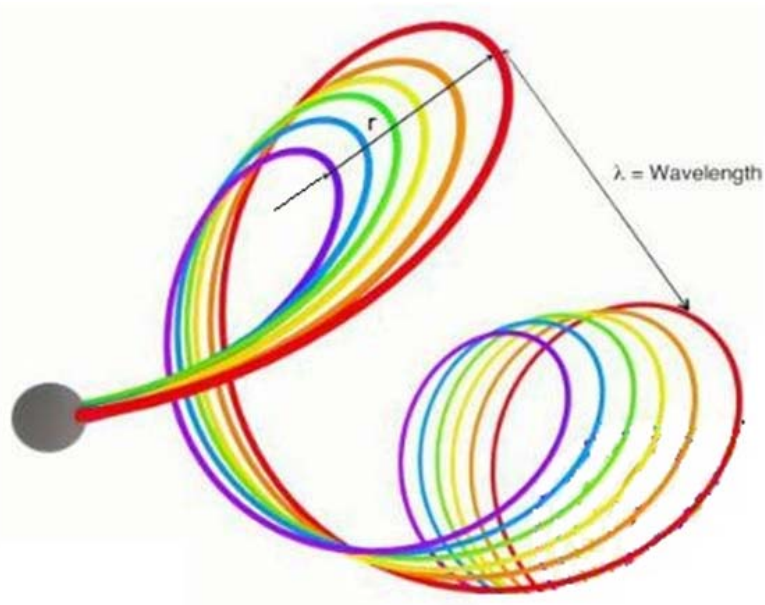

Fig. 4. Diagram of different gyroradius during a photon's trajectory around an imaginary axis.

We assume that the mass of the photon is $m=$ $1.637 \times 10^{-35} \mathrm{~kg}$ and that the approximate value of its angular velocity is equal to $=1.67 \times 10^{15} \mathrm{rad} / \mathrm{s}$. The gyroradius can be calculated for three spectra;

Given the mass value of a photon and $\omega$, the gyroradius for the visible spectrum should be between $100 \mathrm{~nm}$ and $150 \mathrm{~nm}$ (Fig.5).

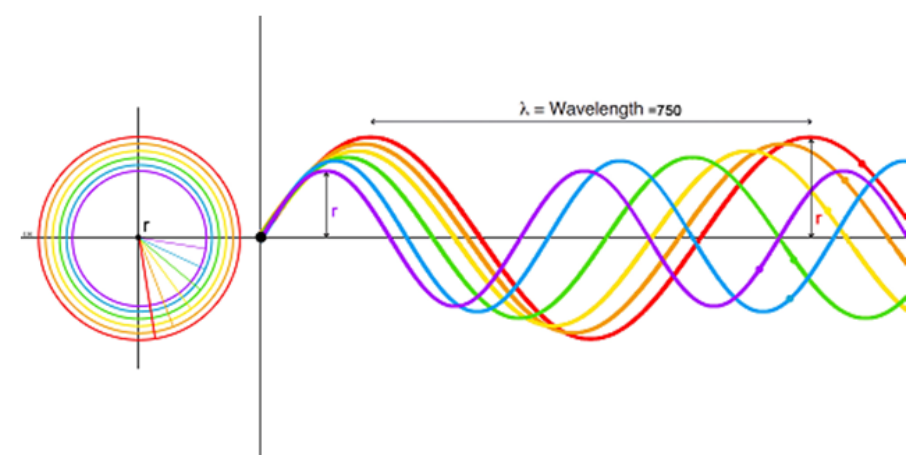

Fig. 5. Diagram of the color Variety of Photons.

\section{References}

1. D. Harrison, Complementarity and the Copenhagen Interpretation of Quantum Mechanics, UPSCALE, Dept. of Physics, U. of Toronto, (2002)

2. G.R. Fowles, Mechanics of Rigid Bodies: Planar Motion, Analytical Mechanics, $7^{\text {th }}$ edition, (London, U. K: THOMSON, 1987)

3. S. Gasiorowicz, The limits of Classical Physics, Quantum Physics, (New York, NY, USA: JOHN WILEY\&SONS, 1974)

4. G.R. Fowles, Newtonian Mechanics: Rectilinear Motion of a Particle, Analytical Mechanics, $7^{\text {th }}$ edition, (London, U. K: THOMSON, 1987) 
5. R.T. Weidner, R. Sells, Relativistic Dynamics: Momentum and Energy, Elementary Modern Physics, $4^{\text {th }}$ edition, (New York, NY, USA: Allyn and Bacon, 1976)

6. D. Klinghardt, The Neurophysiology of light, Journal of Optometric Phototherapy, 35-40, (2003)

7. M.W. Zemansky, R.H. Dittman, Heat and the First Law of Thermodynamics, Heat and Thermodynamics, $7^{\text {th }}$ edition, (New York, NY, USA: McGraw-Hill, 1997)
8. D. Haliday, R. Resnick, J. Walker, wave II, Fundamentals of physics, $5^{\text {th }}$ edition, (New York, NY, USA: JOHN WILEY\&SONS, 1997)

9. D. Haliday, R. Resnick, J. Walker, Potential Energy and Conservation of Energy, Fundamentals of physics, $5^{\text {th }}$ edition, (New York, NY, USA: JOHN WILEY\&SONS, 1997). 